\title{
Land plant molecular phylogenetics: a review with comments on evaluating incongruence among phylogenies
}

Cymon J. Cox - Centro de Ciências do Mar, Universidade do Algarve, Faro 8005-139, Portugal.

\begin{abstract}
Land plants evolved from freshwater charophyte algal ancestors during a single transition to the terrestrial environment. The six major lineages of land plants are divided into two groups, the bryophytes (liverworts, mosses, and hornworts) and the tracheophytes (lycophytes, ferns, and seed plants), but while the tracheophytes are thought to be monophyletic, the bryophytes have typically been considered as the direct ancestors of tracheophytes and therefore an artificial, nonmonophyletic, group. Here the molecular phylogenetic evidence for relationships is reviewed and evaluated especially in-light of large genome-level studies that have been completed in the last few years. Consideration is given to how to evaluate competing hypotheses with respect to the underlying evolutionary assumptions of the models used to analyse the molecular data, and the degrees of support for particular hypotheses. It is concluded that currently the two most-favourable hypotheses are that the bryophytes are a monophyletic group, or that a lineage consisting of liverworts and mosses branched first among land plants with the hornworts the most-closely related lineage to tracheophytes. Although hitherto rarely considered, the possible monophyly of bryophytes has important implications for the morphological reconstruction of the last common ancestor of all land plants. Indeed, it might suggest that the ancestor of land plants was vascularised and had alternating generations that were more isomorphic than is found in extant taxa. The evolution of the bryophytes might then have proceeded through elaboration of the gametophyte and reduction of the sporophyte, while the opposite being true of the tracheophyte lineage.
\end{abstract}




\section{Introduction}

The origin and diversification of terrestrial plants is one of the most important events in Earth history, but it has also proven to be one of the most challenging for phylogenetic systematics to reconstruct. Similarities between reproductive features of land plants, such as the possession of motile male gametes, and comparable cellular structures and pigmentation between land plants and algae, led as early as the late $19^{\text {th }}$ century to the widely-held belief that land plants were likely derived from a single green algal ancestor (Kenrick and Crane 1997). Similarly, the tracheophytes, namely the seed plants, ferns, and lycophytes, a group with a conspicuous and dominant sporophyte generation that has a vascular system to transport water and metabolites through the plant body, were also recognised very early as a natural evolutionary group (figure by Haeckel 1876 depicted in Kenrick and Crane 1997). In today's terms, such a group is described as monophyletic and consists of all the descendants of a common ancestor. However, the monophly of a group comprising the other three major lineages of land plants, namely the gametophytedominant bryophytes comprising the hornworts, mosses, and liverworts, has never been accorded much credibility. From a historical perspective this may be a reflection of the observation that bryophytes share with freshwater algae a similar haploid-dominant alternation of generations, which enforces the view that the "lower" bryophytes are more primitive than the "higher", and implicitly more evolutionarily advanced, tracheophytes (Bower 1890). Although such an evolutionarily simplistic view of constantly increasing complexity is now widely discredited besides which bryophytes are themselves complex and not in any way simplistic despite their small stature - the bryophytes are still most often regarded as the direct ancestors of tracheophytes, although the exact timings of the bryophyte diversification events is a matter of great contention.

The streptophyte green plants as a group include the terrestrial plants that develop via an embryo (embryophytes) and the freshwater algae (charophytes) from which they are descended (Palmer et al. 2004). Indeed, recognition that the embryophytes evolved from charophyte algae 
settled a long-standing debate concerning the evolution of alternating generations in land plant lifecycles (for a recent review see Haig 2008). Charophyte algae have haplontic life-cycles with zygotic meiosis, suggesting that the diploid multicellular sporophyte generation of the embryophytes had evolved from interpolation of mitotic divisions after zygote formation: an observation that accords with the "antithetic theory" and contrasts with the "homologous theory" whereby the embryophyte ancestor had isomorphic, free-living, generations (Bennici 2008). However, recent molecular phylogenetic analyses (Turmel et al. 2006; Karol et al. 2010; Wodniak et al. 2011, Timme et al. 2012; Civáň et al. 2014, Wickett et al. 2014) have determined that the aflagellate, conjugating charophytes (Zygnematales) that are most often found as single cells, cellular colonies, or filaments, are the most-closely related group to embryophytes. This result is perhaps surprising given that the more-distantly related stonewort charophytes (Charales) are morphologically more complex, with a multicellular branching structure reminiscent of land plants and a suite of cellular characters similar to embryophytes (Karol et al. 2001; McCourt et al. 2004). This finding probably reflects a loss of many of these characters in Zygnematales as a consequence of their evolving conjugation as a means of reproduction (Timme et al. 2012).

It is now well-established that there was a single evolutionary transition to embryophyte land plants from an aquatic charophyte algal ancestor (e.g. Karol et al. 2001), however, the relationships among the major groups of embryophytes have proven difficult to determine. Embryophyte diversity is divided into two major groups, the bryophytes and the tracheophytes (also called vascular plants), each of which has three major divisions which represent monophyletic lineages, namely, the Bryophyta (mosses), Marchantiophyta (liverworts), and Anthocerotophyta (hornworts), and the Lycopodiophyta (lycophytes), Moniliformopses (ferns), and Spermatophyta (seed plants), respectively (Figure 1). The tracheophytes all have a diploiddominant life-cycle with a vascularised sporophyte and typically form a statistically well-supported monophyletic group (they share a single common ancestor that is unique to them) in phylogenies. By contrast, the three bryophyte groups have haploid-dominant life-cycles where the unbranched, determinate sporophyte remains attached to, and is nourished by, the gametophyte. Despite these similarities in life-cycle, the three bryophyte lineages have most often been considered 
"independently evolving" lineages that likely do not form a monophyletic group (Crandall-Stotler 1980).

An ancestral nature of bryophytes was supported by the earliest formal phylogenetic analyses of land plants conducted by Parenti (1980), who established that bryophytes were paraphyletic (that is, a group with a common ancestor but not all the descendants of that ancestor) based on morphological characters, with mosses and liverworts grouped together, but with hornworts most closely related to the vascular plants. By contrast, in a similar morphological cladistic analysis by Mishler and Churchill (1986), the liverworts alone were resolved as the earliest-branching lineage, with mosses most closely related to the tracheophytes, and the hornworts branching intermediately. These pioneering analyses in many ways confirmed previous assessments of land plant evolution based on less formal methods and cemented the idea of the bryophyte plant form as being ancestral to the more "advanced" vascular plants of the tracheophytes. Indeed, the seemingly elegant progression of increasing complexity in plant form, through single-celled algae to mulicellular thallose algae, to bryophytes, and then tracheophytes, is still commonly believed to be correct, although the exact details have changed.

Newer hypotheses may necessitate a re-think as to the earliest divisions among land plants and what form the ancestral land plants took. The novel, if slightly controversial at the time, morphological insight of Mishler and Churchill was that the conducting tissue (leptoids and hydriods) of mosses was homologous to the vascular tissue found in the tracheophytes (Mishler and Churchill 1986), and although this homology has since been challenged (Ligrone et al. 2000; Renzaglia et al. 2007), developmental genetics has also identify these tissues as homologous (Xu et al. 2014). Indeed, analyses of morphological and developmental characters have consistently supported a non-monophyletic bryophytes from which vascular plants are derived (Mishler and Churchill 1986; Bremer et al. 1987; Mishler et al. 1994; Garbary and Renzaglia 1998; Renzaglia et al. 2000), as have analyses that have included fossil plant taxa (Kenrick and Crane 1991; Kenrick and Crane 1987) and those employing supra-sequence genomic-level characters (Kelch et al. 2004; Mishler and Kelch 2004; Civáň et al. 2014). The only exceptions to this have been the analyses of ultrastructural traits of male gametogenesis which tend to resolve a monophyletic 
bryophyte clade (monophyletic group), with mosses more closely related to liverworts than hornworts (Garbary et al. 1993; Renzaglia et al. 2000).

\section{Molecular phylogenetics}

The relative value of using morphological or molecular characters for the reconstruction of phylogeny have been debated vigorously, with little resolution or conclusion (Hillis 1987; Baker et al. 1998). While each data type has merits and demerits, it is undeniable that in a time where genomic DNA sequence data are so easily obtained, the focus of phylogenetic tree reconstruction has shifted almost wholly to the molecular realm. Nevertheless, ultimately a molecular phylogenetic tree in itself is of little value except for its ability to classify and predict change in an evolutionary framework, and its use to develop hypotheses of organismal trait evolution.

As with morphological analyses, the majority of molecular phylogenetic analyses of land plants have supported the non-monophyly of the bryophytes, although molecular-based trees have tended to place the liverworts alone as the earliest-diverging taxon (e.g. Lewis et al. 1997), a hypothesis that has come to be the most widely accepted. Despite this trend, almost all possible combinations of relationships among the three bryophyte groups and tracheophytes have been published in molecular phylogenetic trees (Table 1), including ones where the bryophytes are a monophyletic group (e.g. Nishiyama et al. 2004). The difficulty in determining the relationships among the six embryophyte groups is likely due in part to their rapid lineage diversification, perhaps as a response to niche adaptation, soon after terrestrial colonisation, leaving little time for accumulation of distinguishing characters (synapomorphies) to arise in early-diverging lineages (Karol et al. 2001; Liu et al. 2014). Such phylogenetic uncertainty is especially problematic as evolutionary analyses of the key genetic and morphological innovations underpinning land colonisation are important to our understanding the ecology of environments and early bioticallydriven terrestrial environmental changes.

Despite the ambiguity among phylogenetic analyses of land plants, it is commonly assumed that bryophytes are paraphyletic and the liverworts are the earliest-diverging taxon (e.g. Bowman et al. 2007; Clarke et al. 2011). However, with the increased availability of data due to advances in 
sequencing technology, as well as advances in phylogenetic modeling and gene tree evolutionary theory, bryophyte relationships have again come under close scrutiny and recent analyses have yet again challenged the widely-held belief of the ancestral nature of bryophytes in land plant history. How then to make sense of conflicting data sets, and even conflicting analyses of the same data? The arguments are often complex and nuanced, as no one aspect of evolutionary analyses is likely to be sufficient and alone necessary to guarantee that the result converges on the true phylogeny. Here I outline some considerations when assessing modern phylogenetic techniques and the results of those analyses.

\section{Evaluating competing phylogenetic reconstructions}

It is tempting to view phylogenetic reconstruction as fraught with difficulty and controversy, given the high-profile debates that exist on the origin of eukaryotes, animals, flowering plants, and of course many other relationships in the tree of Life. But such a view tends to overlook the remarkable advances that have been made: after all, if the phylogenetic evidence is consistent and points toward a certain relationship, it is eventually accepted as theory no matter how previously controversial such a hypothesis might have been. Indeed, the vast majority of phylogenetic relationships appear non-controversial even in land plant evolution: a) land plants are monophyletic and derived from charophyte algae, b) each of the six major land plant groups (mosses, liverworts, hornworts, lycophytes, ferns, and seed plants) are monophyletic, and c) vascular plants are monophyletic, etc. Nevertheless, despite much research, the relationships among land plant groups had proven difficult to reconstruct with any consistency or confidence. Indeed, the origin of land plants has become a pivotal question in phylogenetic systematics, prompting the use of new models and theory (Cox et al., 2014; Wickett et al., 2014) and helping drive the adoption of new methods (Sayyari and Mirarab, 2016).

How then to navigate a sea of competing, and often well supported, analyses? The concept of support for a phylogenetic hypothesis is important but should be seen only in the context of the particular data set being analysed and the assumptions implicit in the model. Here bootstrap support of $>80 \%$, or Bayesian posterior probabilities $>0.95$, are interpreted as being strong and 
statistically significant support. The apparent, though sometimes weak, conflict between ML bootstrap and Bayesian analyses under similar models can be attributed to their different statistical frameworks: while bootstrap analyses measure the long-term probability of obtaining a similar result given additional data of the same kind, Bayesian analyses directly assess the probability of the model given the data (Shoemaker et al. 1999). When different methods and different data converge on a single species tree, we can have good confidence in having reconstructed the real phylogenetic history of the species. More specifically, analyses conducted using different genes and genomes (organellar and nuclear data) and different analytical methods (distance methods, maximum parsimony, likelihood and Bayesian methods) would ideally converge to the same, correct, species tree. However, some relationships are especially difficult to reconstruct, typically because lineage diversification occurred in a relatively short time-span, leaving few changes in support of relationships. In addition, gene (or contiguous non-recombining sequence) trees that conflict with the species tree due to population level processes (e.g. incomplete lineage sorting, hybridisation, introgression) can result in well-supported but incorrect phylogenies (Degnan and Rosenberg 2006 and 2009). Indeed, when faced with competing phylogenetic hypotheses, it may seem tempting to accept the result that is found most frequently as being correct, and ignore contradictory results as mere aberrations. But this type of consensus summarising of multiple analyses is often flawed: when a particular relationship is difficult to reconstruct, it can be easier to find an incorrect tree than it is the true species phylogeny. Indeed, in my view multiple congruent analyses do not out-weigh the importance of one "better" analysis that results in a different tree. The problem is determining which are the most appropriate analyses, and which are inadequate, in the context of some given data.

Until relatively recently, it has been assumed (often due to computational expediency) that the correct tree is most often than not an emergent property of concatenating large, so-called phylogenomic, amounts of data that negate statistical sampling (stochastic) errors (e.g. Rokas et al. 2003; Jeffroy et al. 2006). Indeed, older phylogenetic divergences that occur rapidly in time, that is, deep and short internal branches of the phylogeny, are often very problematic to reconstruct because they retain so few defining substitutions in extant descendants (Whitfield and Lockhart 2007). In such cases these relationships are often accurately resolved by employing larger 
amounts of data. However, the expectation of a positive correlation between increased phylogenetic accuracy and larger data sets is not always met, as longer sequences can also amplify non-historical signals (biases) in the data that can result in systematic errors in tree reconstruction if inadequately accounted for by the substitution model (e.g. Phillips et al. 2004; Rodríguez-Ezpeleta et al. 2007). In the context of maximum parsimony (which implements an implicit model of change - Swofford et al. 1996), these biases lead to inaccurate phylogenies due to homoplasy (convergent similarity) being undetected, and erroneously reconstructed as ancestrally shared characters (synapomorphies) supporting the close relationship of taxa that are in reality more distantly related. For instance, biases in the rate of substitution among lineages can lead to the accumulation of sufficient amounts of homoplasy in fast evolving lineages to reach substitutional "saturation", which overwhelms the historical signal and causes the lineages to be joined by convergent similarity - the often cited phenomenon of "long-branch attraction" (Felsenstein 1978). Although in this case including additional taxa can increase phylogenetic accuracy when they segment long branches in the tree (Baurain et al. 2007; Hedtke et al. 2006), the additional taxa will not be beneficial if biases remain that are not adequately accounted for by the model (Poe and Swofford 1999; Heath et al. 2008). Better sampled trees are therefore not necessarily more accurate than sparsely sampled trees when biases exist, and in fact, additional taxa may introduce, or strengthen, biases not present in the original data (Kim, 1996; Rokas and Carroll 2005; Gatesy et al. 2007). Moreover, adding more data may even be detrimental if its inclusion precludes the use of more realistic models due to increased computational burden. Importantly, with the advent of next-generation sequencing, data is often no longer the limitingfactor and the emphasis in phylogenetics has shifted to constructing better-fitting and more realistic models (Baurain et al. 2007). But this means that although there may be sufficient data available for a specified phylogenetic question, there is still a computational balance to be struck between numbers of taxa, the amount of data, and model complexity that often leads to conflicting results and controversy surrounding difficult-to-determine relationships.

To obtain an accurate phylogenetic result, the evolutionary processes that require modeling can occur at the level of the gene (or smaller non-recombining sequence unit), the locus, and the 
phylogenetic tree. At the level of the sequence data, variation in instantaneous substitution rates among nucleotides (or amino-acids) and their frequency (base composition) - which together specify the substitution rates - can vary across the data and across the tree (i.e. site- and timeheterogeneous processes). Variation in the absolute rate of substitution at sites in the data is commonly modeled by a mixture of rates drawn from a discrete gamma-distribution (Yang 1994), while variation across the branches of the tree is reflected in differing branch lengths. Among sites, variation among types of substitution processes is typically modeled by applying different substitution matrices to different data partitions, be they genes or some other division of the data (e.g. codon position), or by modeling variation in just the base (or amino acid) composition using a non-parametric (where the number of parameters of the model is a priori undetermined) model (i.e. CAT model: Lartillot and Philippe 2004). By contrast, substitution variation across the tree can be modeled by having instantaneous substitution rates (i.e. NDRH model: Foster et al. 2009) or the base composition (i.e. NDCH/NDCH2 model: Foster 2004; nhPhyML: Boussau and Gouy 2006) vary among branches. The older the phylogeny, the more likely it is to have evolved lineagespecific substitution processes, and hence these tree-heterogeneous substitution models are often a better fit to empirical data above the species-level than homogeneous substitution models.

In addition to modeling sequence substitutions, there has in recent years been an increased effort to accommodate processes acting above the level of the sequence in the phylogenetic model hierarchy that can also lead to systematic error (Lartillot 2015). Among loci, population-level variation in the number, fixation, and sorting of alleles can lead to incongruence among species trees and gene (or more specifically locus) trees due to retention and differential sorting of alleles through multiple speciation events, a process referred to as incomplete lineage sorting (ILS). Reconciliation of gene trees and species trees under the assumption of ILS can be achieved by modeling the probability of gene coalescences that are proportional to the effective population size of the branch and its length: large ancestral population sizes and shallow divergences are expected to result in greater species tree-locus tree discordance (Degnan and Rosenberg 2006 and 2009). Although fully hierarchical multi-species coalescent models that estimate both the species tree and gene trees simultaneously are available (e.g. *BEAST: Heled and Drummond 2010), their use is severely limited by their computational complexity, and more often than not, so- 
called "short-cut" methods that start with pre-computed gene trees are used as a convenience. Several implementations of short-cut multi-species coalescent models are available (e.g. MP-EST: Liu et al. 2010; ASTRAL: Mirarab et al. 2014), however, despite their being computationally tractable, simulations have shown that inaccurately computed gene trees are more problematic for these methods than is ILS for concatenation methods (Gatesy and Springer 2014; Patel et al. 2013). Indeed, the statistical consistency of these methods can only be assured in the absence of gene tree estimation error, or if error is present, then only if the tree conforms to a molecular clock (Roch and Warnow 2015), which is very unlikely in phylogenies above the species level. Nevertheless, such assurances are moot, as the length of non-recombining sequence data needed to accurately estimate gene trees is sufficiently long that such sequences are typically an unrealistic expectation of empirical data (Gatesy and Springer 2014; Roch and Warnow 2015). Moreover, for ancient phylogenies where even short internal branches may span such considerable time-scales that the expectation of ILS is greatly diminished due to allele fixation during that period, consequently the use of short-cut multi-species coalescent models for reconstructing ancient phylogenies has been strongly criticised (Gatesy and Springer 2014). Assuming the absence of confounding deep gene coalescences, gene trees can differ from species trees due to gene duplication, differential loss in subsequent lineages, and lateral gene transfer (Szöllősi et al.2014). "Duplication-loss“ (DL) models accounting for these processes are now available but, again, they are computationally expensive in their fully hierarchical implementations that assess both gene tree and species trees simultaneously (Szöllősi et al. 2014). The fundamental difference between ILS and DL models is that gene tree-species tree discordance due to ILS occurs using completely orthologous gene sequences, while DL models account for the same conflict among orthologous and parologous genes.

As a consequence of these varying degrees of model complexity, evaluating competing phylogenetic hypotheses can be challenging. Putting aside the need for adequate sampling of both taxa and data, the goodness-of-fit of a set of candidate models for a particular data set can be assessed by computing a hierarchical likelihood ratio test (Posada and Crandall 1998) or by calculating Bayes factors in a Bayesian statistical setting (Nylander et al. 2004). However, there are currently no available models that are able to account for all known processes affecting 
phylogenetic reconstruction - both data- and time-heterogeneous processes. At present, for a specific phylogenetic question we are often presented with an array of competing hypotheses based on different data and implementing a variety of different models. Thus analyses must be viewed in light of the data and model that they use, and the substitution and tree-evolutionary processes that are known to be present in the data and that are accounted for, and those that are not. For instance, if an analysis uses a site-homogeneous composition model and yet the data have not been shown to be composition homogeneous, then the extent to which the reconstruction is affected by potentially heterogeneous compositions is unknown, and weakens the credibility of the analysis. Likewise, concatenation methods that assume orthology among sequences ignore, due to their presumed absence, the tree-evolutionary processes of gene duplication and loss, and lateral gene transfer (and perhaps ILS, depending on the tree depth) and would naturally be less credible than models that accounted for these processes if the sequences were not orthologous. We might not reject a concatenated gene analysis that does not demonstrate that the genes are congruent, but we should be wary of assuming congruence if the analyses do not demonstrate as much, and our confidence in the analysis should be duly adjusted. In practice then, to gauge the value of a phylogenetic hypothesis we need to be able to evaluate the extent to which the model fits the data at hand - be it DNA, amino-acids, or some partition of such data - both with regards to known properties of that data, and to those properties which remain unassessed.

\section{Current phylogenetic hypotheses of land plants}

Table 1 is a list of formal phylogenetic studies that use explicit cladistic or molecular evolutionary models. Included are only studies with trees published in the main article that fully resolve relationships (the lineage or taxon has only one sister-group and is not placed at a polytomy) among the three bryophyte groups with respect to the tracheophytes, and where each group was monophyletic or consisted of a single taxon. Consequently, many analyses where only two bryophyte groups are included, even if their relationships are well supported, or where the three bryophyte lineages remain only partially unresolved (e.g. Malek et al. 1996), were not considered. However, studies are included even if relationships were not statistically supported, as high 
statistical support is often not possible with a restricted number of morphological characters, and even high support can still be obtained for the wrong tree if systematic biases are present in molecular analyses. In Table 1 competing topologies are given names that typically describe the branching of bryophyte groups, using the common used terminology of "basal" groups. It should be noted that the term "basal" has no topologically or evolutionary meaningful usage and typically identifies the "smallest earliest-branching group sister to the rest of the clade" - liverworts in a "liverworts-basal" tree are no more "basal" to the rest of the land plants, than are the rest of the land plants "basal" to liverworts - it is just conventional usage, and it is used here for convenience.

Table 1 contains 44 unique studies, some which presented more than one main hypothesis. Perhaps the most striking aspect of the table is the number of different hypotheses generated. For five taxa - in this case, an algal outgroup, the three bryophytes groups, and the tracheophyte lineage - there are 15 possible unrooted topologies: of those a remarkable nine different hypotheses of land plant relationships have been published in studies. The first molecular study, based on 5S rDNA sequences (Hori et al. 1985), was published five years after the first morphology study (Parenti 1980). During the next decade and a half, an additional four studies of general morphology (Bremer 1985; Mishler and Churchill 1986; Bremer et al. 1987; Kenrick and Crane 1987), and four studies of male sperm ultrastructure (Garbary et al. 1993; Mishler et al. 1994; Garbary and Renzaglia 1998; Renzaglia et al. 2000) were published. Since the year 2000 no further morphological studies have been undertaken, although structural features of the plastid genome have been used (Kelch et al. 2004; see also Mishler and Kelch 2009; Civán̆ et al. 2014). Morphological characters support either a "liverworts-basal" topology (Parenti 1980; Bremer 1985; Mishler and Churchill 1986; Mishler et al. 1994) or a "hornworts-basal" topology (Bremer et al. 1987; Mishler et al. 1994; Renzaglia et al. 2000). By contrast, studies of sperm ultrastructural characters almost always resolve "monophyletic bryophytes" (Garbary et al. 1993; Mishler et al. 1994; Garbary and Renzaglia 1998; Renzaglia et al. 2000), with a single exception in a study where "hornworts-basal" was resolved (Garbary and Renzaglia 1998). The analysis of plastid structural characters resolved a "liverworts-basal" topology (Kelch et al. 2004). 
Molecular studies of land plants are far more numerous that those of morphology; 44 as compared to 10 studies. Inevitably it is difficult to summarise an already selective set of studies and find commonalities when so many variables influence phylogenetic reconstruction, e.g. taxon sampling, sequence data characteristics, model complexity, and methods. However some general trends are apparent even if exceptions can be found to all: 1) plastid amino-acids tend to support a monophyletic bryophytes (Nishiyama et al. 2004; Rodríguez-Ezpeleta et al. 2006; Lemieux et al. 2007; Cox et al. 2014; Civáň et al. 2014; Wickett et al. 2014; Puttick et al. 2018; Gitzendanner et al. 2018), 2) plastid nucleotides tend to support paraphyletic bryophytes, especially "liverwort, moss, hornwort" (Lewis et al. 1997; Delwiche et al. 2002; Nishiyama et al. 2004; Wolf et al. 2005; Smith et al. 2009; Gao et al. 2010; Chang and Graham 2011; Fiz-Palacios et al. 2011; Magallón et al. 2013; Kim et al. 2014; Ruhfel et al. 2014; Zhong et al. 2014), or 3) plastid nucleotides support "moss plus liverwort-basal" with hornworts sister to tracheophytes (Nishiyama et al. 2004; Turmel et al. 2006; Lemieux et al. 2007; Qiu et al. 2006; Gao et al. 2010; Karol et al. 2010), and 4) the results of nuclear rDNA studies depend on the molecule, with 5S rDNA supporting "monophyletic bryophytes" embedded in a paraphyletic tracheophytes (Hori et al. 1985; Steele et al. 1991; Quandt et al. 2004), and 18S (and or 26S) supporting "hornworts-basal, moss plus liverworts" (Hedderson et al. 1996, 1998) or "liverworts-basal, moss plus hornwort" (Waters et al. 1992; Mishler et al. 1994). Few analyses of nuclear and mitochondrial data sets have been conducted, but neither data type gives entirely consistent results (e.g. Duff and Nickrent 1999; Wickett et al. 1014; Liu et al. 2014). Combining various data sources has also led to inconsistent results, for instance analyses of nuclear, mitochondrial, and plastid nucleotides and nuclear rDNA (Nickrent et al. 2000) resulted in "hornworts-basal, moss plus liverworts" while combined plastid and mitochondrial nucleotides, and nuclear, plastid and mitochondrial rDNA resulted in "liverworts, moss, hornwort" (Qiu et al. 2006).

Typically, more recent studies have contained better taxon selections, larger quantities of sequence data, and employed more complex models or methods, but not always. Since 2010 , ten plastid (49 loci - Karol et al. 2010, reanalysed by Cox et al. 2014; 79 loci - Gao et al. 2010; 83 loci Civán̆ et al. 2014; 78 loci - Ruhfel et al. 2014; 72 loci - Zhong et al. 2014; 35 loci - Kim et al. 2014; 79 and 88 loci - Lemieux et al. 2014, 2016; 78 loci - Gitzendanner et al. 2018), two mitochondrial 
(40 loci - Turmel et al. 2013; 41 loci - Liu et al. 2014), and two nuclear studies (674 loci - Wickett et al. 2014, reanalysed by Puttick et al. 2018) have been published with data sets containing more than 20 loci (which here are arbitrarily considered to be "genome-level" studies). Five studies of plastid nucleotide data have resulted in topologies that favour either "liverwort, moss, hornwort" and "liverwort plus moss-basal" (Gao et al. 2010; Karol et al. 2010; Ruhfel et al. 2014; Zhong et al. 2014; Kim et al. 2014). However, Cox et al. (2014) demonstrated that the support for nonmonophyletic bryophytes in the study of Karol et al. (2010) (and in the study of plastid nucleotides in Qiu et al. 2006) was a result of a systematic bias in composition among lineages due to synonymous substitutions, and moreover, the authors suggested that this bias is potentially a common problem in any analysis of protein-coding nucleotides where synonymous substitutions occur at rates orders of magnitude greater than those of non-synonymous substitutions. Consequently, the authors argued that the analyses of amino acids that resulted in "monophyletic bryophytes" should be preferred (see also Civáň et al. 2014). Not all genomic-level analyses of plastid proteins, however, support "monophyletic bryophytes"; two analyses resolve "liverwort plus moss-basal" (Ruhfel et al. 2014; Lemieux et al. 2016), and one "moss, liverwort, hornwort" (Lemieux et al. 2014). Strong statistical support for non-monophyly of bryophytes was lacking (i.e. $54 \%$ ) in the study of Ruhfel et al. (2014), but in the two other studies strong support was obtained for the sister-group relationship of hornworts and tracheophytes (89\% bootstrap support under a $\mathrm{GTR}+\Gamma_{4}$ model and 1.0 posterior probability under a CATGTR $+\Gamma_{4}$ (there was no support for mosses-basal) - Lemieux et al. 2014; 1.0 posterior probability under a CATGTR $+\Gamma_{4}$ model Lemieux et al. 2016). By contrast, analyses of similar data resulted in "monophyletic bryophytes" (1.0 posterior probability, under both gcpREV $+\Gamma_{4}$ and gcpREV $+\mathrm{I}+\Gamma_{4}+\mathrm{NDCH}-\mathrm{Cox}$ et al. (2014) (gcpREV is an empirical matrix specifically for green plants; Cox and Foster 2013)), and CAT+gcpREV $+\Gamma_{4}$ (0.89 posterior probability - Civáň et al. 2014). Besides differences in taxon complements, the main difference between these analyses is the use of an empirical substitution matrix (gcpREV) versus estimation of the rates directly from the data (GTR) - in theory, the latter fits the data better as the substitution rates are estimated directly from the data at hand. So although there is good reason to believe the support from plastid nucleotide data for a "hornworts plus tracheophytes" clade is due solely to synonymous substitutions that accumulate composition 
biases leading to systematic error, one of the current best-fitting amino-acid model (CAT) results in support for hornworts sister to tracheophytes over a "monophyletic bryophytes" tree. What remains to be tested is whether modeling among-site composition bias (i.e. CAT model) and modeling among-lineage composition bias (i.e. NDCH model) simultaneously reconstructs the bryophytes as paraphyletic or monophyletic.

Despite this impasse, the plastid analyses of Cox et al. (2014) and Lemieux et al. (2014 and 2016) contained only limited numbers of bryophytes - five, three, and four respectively, due to computational limitations. Additional taxa could help to reduce branch lengths and aid resolution among the groups. Indeed, it has been argued that analyses with large taxon complements are a indispensable test for accurate phylogenetic reconstruction when using sparsely sampled but datadense analyses from genome-level studies (Qiu et al. 2006). However, such a proposition would only be useful if the results of studies with large taxon complements were the final arbiter of conflicting phylogenetic hypotheses: an argument I would strongly reject. For instance, the phylogenetic analyses of Qiu et al. (2006) were explicitly aimed at resolving land plant relationships, and in one data set contained 193 taxa for five loci (two plastid protein-coding genes, two plastid rRNA genes, one mitochondrial rRNA gene, and one nuclear rRNA gene). The resulting topology strongly supported the "liverworts-basal" hypothesis (Fig. 1, Qiu et al. 2006). However, the same result can be obtained, albeit with slightly lower support values, from the same data with similar methods but using only 27 taxa (14 land plants; six bryophytes, eight tracheophytes) when taxa are chosen to sample both lineages from the first divergence in each of the three bryophyte lineages (Fig S.21 Cox et al. 2014). Consequently it is not large taxon numbers per se that are necessary but "judicious" sampling that is relevant to the question, and especially sampling either side of the first divergence of study clades, to reduce branches between major clades to a minimum. Indeed, if your question is land plant relationships, then including large numbers of taxa in the major clades may not be the optimal sampling strategy if model complexity is necessarily limited by computing times and superfluous taxa introduce additional biases.

While nuclear ribosomal gene data has a long tradition of usage in land plant phylogenetics (Hori et al. 1985; Steele et al. 1991; Waters et al. 1992; Hedderson et al. 1996 and 1998; Nishiyama and Kato 1999; Nickrent et al. 2000), until recently the use of nuclear protein-coding 
genes has been limited to a single study of a Class III HD-Zip gene (Floyd et al. 2006) which support the as-then novel "moss, hornwort, liverwort" phylogeny. However, nuclear protein-coding genes came to the fore when Wickett et al. (2014) published analyses of 852 nuclear genes for 103 land plants: a pioneering study far exceeding the numbers of genes available from plastids. Nevertheless, despite the extensive data set, analyses of the data provided conflicting hypotheses. The many data treatments and analyses provided varying results and levels of support, but the authors chose to highlight two results: firstly, ML analyses of first and second codon position nucleotides, which supported "hornworts-basal", and secondly, short-cut coalescent analyses of amino-acid gene trees which supported "monophyletic bryophytes". In general, as with plastid data, analyses of nucleotides supported a non-monophyletic bryophytes whereas amino-acid data supported bryophyte monophyly, although again there were many exceptions. However, strong support for the hornworts-basal topology when using $1^{\text {st }}$ and $2^{\text {nd }}$ codon position nucleotides can be attributed solely to synonymous substitutions (at ARG, LEU, and SER codons) at these sites (Sousa and Cox, unpublished) as was similarly observed in plastid data (Cox et al. 2014). Reanalysis of these data by Puttick et al. (2018), using a better-fitting site-heterogeneous composition model (CAT) and branch-heterogeneous composition model (NDCH2; Foster 2004), also supported "monophyletic bryophytes", but other less well-supported analyses found some support for either "hornwort-basal" or "liverwort plus moss-basal" (Puttick et al. 2018). The strength of support for the liverwort plus moss clade in their analyses, as well as congruence with many other analyses that recover the same clade, led the authors to formally name the clade "Setaphyta": the name recognises that both mosses and liverworts have a sporophytic seta (although developmentally different) which is absent in hornworts.

Analyses of mitochondrial protein-coding genes are similarly limited to two recent studies. CAT-model analyses by Turmel et al. (2013) of the nucleotide positions (identified after removal of the fastest-evolving $20 \%$ of sites) of 40 mitochondrial gene resulted in a "liverwort, moss, hornwort" tree, however while support for hornworts sister to tracheophytes was strong, the support for liverworts-basal, as opposed to liverworts plus mosses, was considerably weaker. In analyses by Liu et al. (2014), strong support for "mosses-basal" from nucleotide analyses of 41 protein-coding genes was again shown to be due to among-lineage composition bias, a similar observation to 
those of Cox et al. (2014) for plastid nucleotides. However, strong support was obtained for hornworts as the sister group to tracheophytes, although the absence of ferns, one of the major lineages of tracheophytes, and the zygnematales, the closest outgroup, from the analyses may have been detrimental to the accuracy of the results.

\section{Conclusions}

In my view there are currently two supported hypotheses of land plant relationships: firstly "monophyletic bryophytes", and secondly "liverworts plus mosses-basal", with hornworts sister to tracheophytes (Fig. 1). Both of these hypotheses assume that mosses and liverworts are sister taxa, a hypothesis which is strongly supported by most recent genome-level phylogenetic analyses and validates their recognition as a formal group, the Setaphyta. These two hypotheses are favoured because of two suppositions drawn from the arguments laid out in the preceding discussion: 1) that analyses of slower evolving amino-acids are to be preferred over those of nucleotides, which due to their rate of change accumulate larger amounts of homoplasy at the deep phylogenetic level of land plant diversification ( 450-480MYA), and 2 ) better-fitting models, especially those that account for composition heterogeneity either among lineages or among sites, tend to support (or at least not conflict with) these hypotheses over those that favour either one of the bryophyte groups alone as the earliest-diverging lineage. For analyses of both plastid and nuclear protein-coding gene nucleotide sequences, it can be shown that conflict with amino-acid translations of the same data is a result of biases introduced by synonymous substitutions. Moreover, phylogenies of non-synonymous substitutions tend to be congruent (or not conflicting) with amino-acid trees, and indeed, analyses of both plastid and nuclear gene proteins support the monophyly of bryophytes using some composition heterogeneous models (NDCH and CAT). The important exception here is, however, the CAT+GTR plastid protein analysis, which supports "liverwort plus moss-basal". Mitochondrial protein data also strongly support a similar topology; but these data are limited by the smaller number of genes and currently are the most poorly sampled taxonomically (lacking major plant lineages). It is important to note that both organellar genomes, the plastid and mitochondrion, are thought only rarely to undergo recombination and are therefore 
likely single linkage groups and representative of single gene trees, although they are not often conceptually treated as such. With this in mind, although there is conflicting evidence, especially between the mitochondrial data and the nuclear plus plastid data, the results from the mitochondrial analyses are less convincing, and therefore the monophyly of bryophytes should be considered the better supported hypothesis at this time.

Land plant phylogeny is crucial to our understanding the development of plant form and life history, as well determining the place of plants in the paleo-environment. It seems evident, given the analytically short branches separating clades in phylogenies, and consequently the difficulty in reconstructing relationships, that the four major lineages, liverworts, mosses, hornworts, and tracheophytes, evolved over a relatively short evolutionary time-span, with lineages accumulating few diagnostic changes. It seems plausible that once the charophyte algal ancestor of land plants had evolved the necessary form and physiology to survive out of water, for instance tolerance to UV and the mechanisms to maintain cell turgor pressure, a panorama of ecological niches became available for exploitation, which ultimately drove a relatively rapid diversification of new lineages. The paucity of bryophyte macro-fossils before the Silurian-Devonian boundary ( 420MYA) when tracheophytes are first recorded has vexed botanists, because of the obvious implication of phylogenies that indicate tracheophytes are derived from bryophytes. Indeed this absence has been attributed to the poor preservation attributes of bryophytes, a group that do not unequivocally appear in the fossil record until much later (liverworts 393MYA; mosses 100MYA; hornworts 34MYA - Clarke et al. 2011); although some Cambrian ( 510MYA) trilete spores, and "cryptospores" first occurring in the mid-Ordivician ( 470MYA), are described as bryophyte-like (though they are equally tracheophyte-like as well), thereby filling the void left by the absence of evidence of bryophyte meso-fossils. Modern phylogenetics does, however, pose an alternative explanation: if bryophytes are monophyletic (or in fact even if they are paraphyletic, with liverworts and mosses-basal) they may have evolved much later than assumed and diversified alongside the early tracheophytes. Moreover, the possible monophyly of bryophytes has important implications for the morphological reconstruction of the last common ancestor of all land plants, and by extension the gestalt body-plan needed to recognise early stem-lineage land plants in the fossil record. For instance, if, as now seem likely, the water conducting vessels of bryophytes (leptoids 
and hydriods) are developmentally homologous to xylem and phloem (Xu et al. 2014), and given that there is now very strong evidence mosses and liverworts are sister-groups, it seems likely that all embryophytes are descended from a vascularised most-recent common ancestor of land plants, unless hornworts, which lack specialised conducting vessels, are the earliest diverging lineage (a hypothesis that is not well supported). This would imply that the vascular system in mosses, and liverworts such as Pallavicinia and Jensenia (Crandall-Stotler pers. comm.; Ligrone et al. 2002), are likely reduced to-a-degree from that of the most-recent common land plant ancestor, and if bryophytes are monophyletic that hornworts have likely lost vascularity entirely through reduction. This in turn, raises the intriguing possibility that plant fossil assemblages such as the Rhynie Chert $(\sim 407 \mathrm{MYA})$ are indicative of stem-group land plants from which both bryophytes and tracheophytes could have evolved, perhaps from taxa that had isomorphic generations and from which bryophytes evolved by elaboration of the gametophyte, and tracheophytes via elaboration of the sporophyte, generation. Of course, bryophyte monophyly does not preclude the original explanation that assemblages such as the Rhynie Chert are purely stem-tracheophytes (Kenrick 2017) and that bryophytes have an older history obscured by lack of fossil preservation or our ability to distinguish early bryophytes, but currently the best-supported phylogenetic hypotheses of land plants are not incompatible with a range of novel interpretations of the fossil record.

\section{Acknowledgements}

I would like to thank Laura L. Forrest, Filipe de Sousa, and Matt Johnson for their helpful comments and criticism. This study/work received national funds through FCT - Foundation for Science and Technology through project PTDC/BIA-EVF/1499/2014. This work received national funds through FCT - Foundation for Science and Technology through project

CCMAR/Multi/04326/2013. 
Table 1. Phylogenetic studies of land plant relationships. Analyses are only included if all three bryophyte groups are represented, each is monophyletic if comprising multiple taxa, and all three bryophyte groups are fully resolved among themselves but not necessarily statistically supported. (Modified from Puttick et al. 2018)

\begin{tabular}{|c|c|c|c|}
\hline & Embryophyte sister-group $^{10}$ & Data & Reference \\
\hline \multirow{16}{*}{$\begin{array}{l}\text { "bryophyte } \\
\text { monophyly" }\end{array}$} & Charales & Nuclear rDNA ${ }^{11}$ & Hori et al. 1985 \\
\hline & Charales & Nuclear rDNA $^{11}$ & Steele et al. 1991 \\
\hline & Coleochaetales/Charales & Sperm ultrastructure & Garbary et al. 1993 \\
\hline & Coleochaetales & Sperm ultrastructure & Mishler et al. 1994 \\
\hline & Charales & Sperm ultrastructure & Renzaglia et al. 2000 \\
\hline & charophytes/chlorophytes & Chloroplast amino acids & Nishiyama et al. 2004 \\
\hline & Chlorophyta/Coleochaetales & Chloroplast nucleotides $^{11}$ & Quandt et al. 2004 \\
\hline & Coleochaetales & Chloroplast nucleotides $^{12}$ & Goremykin and Hellwig 2005 \\
\hline & Coleochaetales & Chloroplast amino acids ${ }^{11}$ & Rodríguez-Ezpeleta et al. 2007 \\
\hline & Zygnematales & Chloroplast amino acids and nucleotides ${ }^{11}$ & Turmel et al. 2006 \\
\hline & Zygnematales & Chloroplast amino acids & Lemieux et al. 2007 \\
\hline & Zygnematales & Chloroplast amino acids & Cox et al. 2014 \\
\hline & Zygnematales & Chloroplast amino acids & Civáň et al. 2014 \\
\hline & Zygnematales & Nuclear amino acids & Wickett et al. 2014 \\
\hline & Zygnematales & Chloroplast amino acids & Gitzendanner et al. 2018 \\
\hline & Zygnematales & Nuclear amino acids & Puttick et al. 2018 \\
\hline \multirow{9}{*}{$\begin{array}{l}\text { "hornworts-basal, } \\
\text { moss plus liverworts"2 }\end{array}$} & Coleochaetales & Nuclear rDNA & Hedderson et al. 1996 \\
\hline & Coleochatales/Klebsormidiales & Nuclear rDNA & Hedderson et al. 1998 \\
\hline & Coleochaetales & Sperm ultrastructure & Garbary and Renzaglia 1998 \\
\hline & chlorophytes & Mitochondrial rDNA & Duff and Nickrent 1999 \\
\hline & Chlorophyta/Coleochaetales & Nuclear and chloroplast nucleotides ${ }^{12}$ & Nishiyama and Kato 1999 \\
\hline & Charales/Zygnematales & $\begin{array}{c}\text { Nuclear, mitochondrial, chloroplast } \\
\text { nucleotides and nuclear rDNA }{ }^{12}\end{array}$ & Nickrent et al. 2000 \\
\hline & Coleochaetales & $\begin{array}{c}\text { Morphology, nuclear and mitochondrial } \\
\text { nucleotides } \\
\end{array}$ & Renzaglia et al. 2000 \\
\hline & Zygnematales & Nuclear nucleotides $^{12}$ & Wickett et al. 2014 \\
\hline & Zygnematales & Nuclear amino acids & Puttick et al. 2018 \\
\hline \multirow[t]{2}{*}{ "hornwort, liverwort, } & Charales & Morphology & Bremer et al. 1987 \\
\hline & Coleochaetales & Morphology and nuclear rDNA & Mishler et al. 1994 \\
\hline \multirow[t]{9}{*}{ "liverwort plus moss- } & Coleochaetales & Chloroplast nucleotides $^{13}$ & Nishiyama et al. 2004 \\
\hline & Zygnematales & Chloroplast nucleotides & Turmel et al. 2006 \\
\hline & Zygnematales & Chloroplast nucleotides & Lemieux et al. 2007 \\
\hline & Zygnematales & Chloroplast nucleotides $^{12}$ & Qiu et al. 2006 \\
\hline & Zygnematales & Chloroplast nucleotides $^{12}$ & Gao et al. 2010 \\
\hline & Zygnematales & Chloroplast nucleotides & Karol et al. 2010 \\
\hline & Zygnematales & Chloroplast amino acids & Ruhfel et al. 2014 \\
\hline & Zygnematales & Chloroplast amino acids & Lemieux et al. 2016 \\
\hline & Zygnematales & Nuclear amino acids & Puttick et al. 2018 \\
\hline \multirow[t]{8}{*}{ "liverwort, moss, } & "Charophceae" & Morphology & Parenti 1980 \\
\hline & Coleochaetes/Charales & Chloroplast nucleotides & Lewis et al. 1997 \\
\hline & Charales & Chloroplast nucleotides & Delwiche et al. 2002 \\
\hline & Coleochaetales & Chloroplast genome structure & Kelch et al. 2004 \\
\hline & Coleochaetales & Chloroplast nucleotides ${ }^{12}$ & Nishiyama et al. 2004 \\
\hline & Coleochaetales & Chloroplast nucleotides & Wolf et al. 2005 \\
\hline & Charales/Coleochaetales & Mitochondrial nucleotides & Groth-Malonek et al. 2005 \\
\hline & Charales or Zygnematales & $\begin{array}{l}\text { Chloroplast nucleotide and rDNA, and } \\
\text { mitochondrial and nuclear rDNA }\end{array}$ & Qiu et al. 2006 \\
\hline
\end{tabular}




\begin{tabular}{|c|c|c|c|}
\hline & Charales & $\begin{array}{l}\text { Chloroplast and mitochondrial nucleotides, } \\
\text { and nuclear, chloroplast, and mitochondrial } \\
\text { rDNA }\end{array}$ & Qiu et al. 2007 \\
\hline & Charales & Chloroplast nucleotides & Smith et al. 2009 \\
\hline & Zygnematales & Chloroplast nucleotides & Gao et al. 2010 \\
\hline & Zygnematales & Chloroplast nucleotides & Chang and Graham 2011 \\
\hline & chlorophytes/charophytes & Chloroplast nucleotides and nuclear rDNA & Fiz-Palacios et al. 2011 \\
\hline & Coleochaetales & Chloroplast nucleotides & Magallón et al. 2013 \\
\hline & Charales or Zygnematales & Mitochondrial nucleotides & Turmel et al. 2013 \\
\hline & Charales/Coleochaetales & Chloroplast nucleotides & Kim et al. 2014 \\
\hline & Charales & Mitochondrial nucleotides and amino acids & Liu et al. 2014 \\
\hline & Zygnematales & Chloroplast nucleotides & Ruhfel et al. 2014 \\
\hline & Zygnematales & Chloroplast nucleotides & Zhong et al. 2014 \\
\hline \multirow[t]{6}{*}{ "liverwort, hornwort, } & "charophytes" & Morphology & Mishler and Churchill 1986 \\
\hline & Charales/Coleochaetales & Morphology & Bremer 1985 \\
\hline & Coleochaetales & Morphology & Bremer et al. 1987 \\
\hline & Coleochaetales & $\begin{array}{l}\text { Morphology and chloroplast and nuclear } \\
\text { rDNA }\end{array}$ & Mishler et al. 1994 \\
\hline & Charales/Coleochaetales & Morphology & Kenrick and Crane 1997 \\
\hline & Charales & $\begin{array}{l}\text { Chloroplast and mitochondrial nucleotides, } \\
\text { nuclear rDNA }\end{array}$ & Karol et al. 2001 \\
\hline \multirow[t]{3}{*}{ "liverwort-basal, moss } & Klebsormidiales/Coeleochaetales & Nuclear rDNA & Waters et al. 1992 \\
\hline & Klebsormidiales & Chloroplast and nuclear rDNA & Mishler et al. 1994 \\
\hline & chlorophytes & Mitochondrial rDNA & Duff and Nickrent 1999 \\
\hline \multirow{2}{*}{$\begin{array}{l}\text { "moss, liverwort, } \\
\text { hornwort" } 8\end{array}$} & Charales & Mitochondrial nucleotides & Liu et al. 2014 \\
\hline & Coleochaetales & Chloroplast amino acids & Lemieux et al. 2014 \\
\hline $\begin{array}{l}\text { "moss, hornwort, } \\
\text { liverwort" }\end{array}$ & Charales & Nuclear amino acids & Floyd et al. 2006 \\
\hline
\end{tabular}

${ }^{1}$ (algal outgroup, ((liverwort, moss, hornwort), tracheophytes))

${ }^{2}$ (algal outgroup, (hornwort, ((liverwort, moss), tracheophytes))

${ }^{3}$ (algal outgroup, (hornwort, (liverwort, (moss, tracheophytes))))

${ }^{4}$ (algal outgroup, ((liverwort, moss), (hornwort, tracheophytes)))

${ }^{5}$ (algal outgroup, (liverwort, (moss, (hornwort, tracheophytes))))

${ }^{6}$ (algal outgroup, (liverwort (hornwort (moss, tracheophytes))))

${ }^{7}$ (algal outgroup, (liverwort ((moss, hornwort), tracheophytes)))

${ }^{8}$ (algal outgroup, (moss, (liverwort, (hornwort, tracheophytes))))

${ }^{9}$ (algal outgroup, (moss, (hornwort, (liverwort, tracheophytes))))

${ }^{10}$ either the designated outgroups or, if identified, the most closely related taxon to land plants

${ }^{11}$ note that although bryophytes were monophyletic they were embedded in a paraphyletic

tracheophyte clade 
${ }^{12}$ protein coding-genes $1^{\text {st }}$ and $2^{\text {nd }}$ codon positions only: Goremykin and Hellwig 2005; Nishiyama et al. 2004; Wickett et al. 2014; Nickrent et al. 2000; Gao et al. 2010

${ }^{13}$ a) excluding the nucleotide sites coding leucine and third codon positions, and b) fourfold degenerate sites 
References:

Baker, R.H., Yu, X. and DeSalle, R., 1998. Assessing the relative contribution of molecular and morphological characters in simultaneous analysis trees. Molecular Phylogenetics and Evolution, 9: $427-436$.

Baurain, D., Brinkmann, H. and Philippe, H., 2006. Lack of resolution in the animal phylogeny: closely spaced cladogeneses or undetected systematic errors? Molecular Biology and Evolution 24: 6-9.

Bennici, A., 2008. Origin and early evolution of land plants: Problems and considerations.

Communicative \& integrative biology 1: 212-218.

Boussau, B. and Gouy, M., 2006. Efficient likelihood computations with nonreversible models of evolution. Systematic biology 55: 756-768.

Bower, F.O., 1890. On antithetic as distinct from homologous alternation of generations in plants. Annals of Botany 4: 347-370.

Bowman, J.L., Floyd, S.K. and Sakakibara, K., 2007. Green genes - comparative genomics of the green branch of life. Cell 129: 229-234.

Bremer, K., 1985. Summary of green plant phylogeny and classification. Cladistics 1: 369-385.

Bremer, K., Humphries, C.J., Mishler, B.D. and Churchill, S.P., 1987. On cladistic relationships in green plants. Taxon 36: 339-349.

Chang, Y. and Graham, S.W., 2011. Inferring the higher-order phylogeny of mosses (Bryophyta) and relatives using a large, multigene plastid data set. American journal of botany 98: 839-849. 
Civáň, P., Foster, P.G., Embley, M.T., Seneca, A. and Cox, C.J., 2014. Analyses of charophyte chloroplast genomes help characterize the ancestral chloroplast genome of land plants. Genome biology and evolution 6: 897-911.

Clarke, J.T., Warnock, R. and Donoghue, P.C., 2011. Establishing a time-scale for plant evolution. New Phytologist 192: 266-301.

Cox, C.J., Li, B., Foster, P.G., Embley, T.M. and Civáň, P., 2014. Conflicting phylogenies for early land plants are caused by composition biases among synonymous substitutions. Systematic Biology 63: 272-279.

Cox, C.J. and Foster, P.G., 2013. A 20-state empirical amino-acid substitution model for green plant chloroplasts. Molecular phylogenetics and evolution 68: 218-220.

Crandall-Stotler, B., 1980. Morphogenetic designs and a theory of bryophyte origins and divergence. BioScience 30: 580-585.

Degnan, J.H. and Rosenberg, N.A., 2006. Discordance of species trees with their most likely gene trees. PLoS genetics 2: 68 .

Degnan, J.H. and Rosenberg, N.A., 2009. Gene tree discordance, phylogenetic inference and the multispecies coalescent. Trends in Ecology \& Evolution 24: 332-340.

Delwiche, C.F., Karol, K.G., Cimino, M.T. and Sytsma, K.J., 2002. Phylogeny of the genus Coleochaete (Coleochaetales, Charophyta) and related taxa inferred by analysis of the chloroplast gene rbcL. Journal of Phycology 38: 394-403.

Duff, R.J. and Nickrent, D.L., 1999. Phylogenetic relationships of land plants using mitochondrial small-subunit rDNA sequences. American Journal of Botany 86: 372-386.

Felsenstein, J., 1978. Cases in which parsimony or compatibility methods will be positively misleading. Systematic Zoology 27: 401-410. 
Fiz-Palacios, O., Schneider, H., Heinrichs, J. and Savolainen, V., 2011. Diversification of land plants: insights from a family-level phylogenetic analysis. BMC Evolutionary Biology 11: 341.

Floyd, S.K., Zalewski, C.S. and Bowman, J.L., 2006. Evolution of class III homeodomain-leucine zipper genes in streptophytes. Genetics 173: 373-388.

Foster, P.G., 2004. Modeling compositional heterogeneity. Systematic Biology 53: 485-495.

Foster, P.G., Cox, C.J. and Embley, T.M., 2009. The primary divisions of life: a phylogenomic approach employing composition-heterogeneous methods. Philosophical Transactions of the Royal Society B: Biological Sciences 364: 2197-2207.

Gao, L., SU, Y.J. and Wang, T., 2010. Plastid genome sequencing, comparative genomics, and phylogenomics: current status and prospects. Journal of Systematics and Evolution 48: 77-93.

Garbary, D.J., Renzaglia, K.S. and Duckett, J.G., 1993. The phylogeny of land plants: a cladistic analysis based on male gametogenesis. Plant Systematics and Evolution 188: 237-269.

Garbary, D.J, and Renzaglia K.S., 1998. Bryophyte phylogeny and the evolution of land plants: evidence from development and ultrastructure. In Bryology for the Twenty-First Century, Eds. Bates, J.W., Ashton, N.W., and Duckett, J.G. British Bryological Society, UK. Pp. 45-63.

Gatesy, J., DeSalle, R. and Wahlberg, N., 2007. How many genes should a systematist sample? Conflicting insights from a phylogenomic matrix characterized by replicated incongruence. Systematic Biology 56: 355-363.

Gatesy, J. and Springer, M.S., 2014. Phylogenetic analysis at deep timescales: unreliable gene trees, bypassed hidden support, and the coalescence/concatalescence conundrum. Molecular Phylogenetics and Evolution 80: 231-266. 
Gitzendanner, M.A., Soltis, P.S., Wong, G.K.-S., Ruhfel, B.R., and Soltis, D.E. 2018. Plastid phylogenomic analysis of green plants: A billion years of evolutionary history. American Journal of Botany 105: 291-301.

Goremykin, V.V. and Hellwig, F.H., 2005. Evidence for the most basal split in land plants dividing bryophyte and tracheophyte lineages. Plant Systematics and Evolution 254: 93-103.

Groth-Malonek, M., Pruchner, D., Grewe, F. and Knoop, V., 2004. Ancestors of trans-splicing mitochondrial introns support serial sister group relationships of hornworts and mosses with vascular plants. Molecular Biology and Evolution 22: 117-125.

Haig, D., 2008. Homologous versus antithetic alternation of generations and the origin of sporophytes. The Botanical Review 74: 395-418.

Hedderson, T.A., Chapman, R.L. and Cox, C.J., 1998. Bryophytes and the origins and diversification of land plants: new evidence from molecules. In Bryology for the Twenty-First Century, Eds. Bates, J.W., Ashton, N.W., and Duckett, J.G. British Bryological Society, UK. Pp. 6577.

Hedderson, T.A., Chapman, R.L. and Rootes, W.L., 1996. Phylogenetic relationships of bryophytes inferred from nuclear-encoded rRNA gene sequences. Plant Systematics and Evolution 200: 213224.

Hedtke, S.M., Townsend, T.M. and Hillis, D.M., 2006. Resolution of phylogenetic conflict in large data sets by increased taxon sampling. Systematic Biology 55: 522-529.

Heled, J. and Drummond, A.J., 2009. Bayesian inference of species trees from multilocus data. Molecular Biology and Evolution 27: 570-580.

Hillis, D.M., 1987. Molecular versus morphological approaches to systematics. Annual review of Ecology and Systematics 18: 23-42. 
Hori, H., Lim, B.L. and Osawa, S., 1985. Evolution of green plants as deduced from 5S rRNA sequences. Proceedings of the National Academy of Sciences 82: 820-823.

Jeffroy, O., Brinkmann, H., Delsuc, F. and Philippe, H., 2006. Phylogenomics: the beginning of incongruence? TRENDS in Genetics 22: 225-231.

Karol, K.G., Arumuganathan, K., Boore, J.L., Duffy, A.M., Everett, K.D., Hall, J.D., Hansen, S.K., Kuehl, J.V., Mandoli, D.F., Mishler, B.D. and Olmstead, R.G., 2010. Complete plastome sequences of Equisetum arvense and Isoetes flaccida: implications for phylogeny and plastid genome evolution of early land plant lineages. BMC Evolutionary Biology 10: 321.

Karol, K.G., McCourt, R.M., Cimino, M.T. and Delwiche, C.F., 2001. The closest living relatives of land plants. Science 294: 2351-2353.

Kelch, D.G., Driskell, A. and Mishler, B.D., 2004. Inferring phylogeny using genomic characters: a case study using land plant plastomes. In Molecular Systematics of Bryophytes, Eds., Goffinet, B., Hollowell, V., and Magill, R., Monographs in Systematic Botany from the Missouri Botanical Garden, 98. Missouri Botanical Garden Press, U.S.A. Pp. 3-12.

Kenrick, P., 2018. Changing expressions: a hypothesis for the origin of the vascular plant life cycle. Philosophical Transactions of the Royal Society B: Biological Sciences 373: 20170149.

Kenrick, P. and Crane, P.R., 1997. The origin and early diversification of land plants Kenrick, P. and Crane, P.R., 1997. The origin and early diversification of land plants. A cladistic study (Vol. 560). Smithsonian Institute Press Washington DC. U.S.A. Pp 1-441.

Kim, J., 1996. General inconsistency conditions for maximum parsimony: effects of branch lengths and increasing numbers of taxa. Systematic Biology 45: 363-374.

Kim, H.T., Chung, M.G. and Kim, K.J., 2014. Chloroplast genome evolution in early diverged leptosporangiate ferns. Molecules and Cells 37: 372. 
Lartillot, N., 2015. Probabilistic models of eukaryotic evolution: time for integration. Philosophical Transactions of the Royal Society B: Biological Sciences 370: 20140338.

Lartillot, N. and Philippe, H., 2004. A Bayesian mixture model for across-site heterogeneities in the amino-acid replacement process. Molecular Biology and Evolution 21: 1095-1109.

Lewis, L.A., Mishler, B.D. and Vilgalys, R., 1997. Phylogenetic relationships of the liverworts (Hepaticae), a basal embryophyte lineage, inferred from nucleotide sequence data of the chloroplast gene rbcL. Molecular Phylogenetics and Evolution 7: 377-393.

Ligrone, R., Duckett, J.G. and Renzaglia, K.S., 2000. Conducting tissues and phyletic relationships of bryophytes. Philosophical Transactions of the Royal Society of London B: Biological Sciences 355: 795-813.

Lemieux, C., Otis, C. and Turmel, M., 2007. A clade uniting the green algae Mesostigma viride and Chlorokybus atmophyticus represents the deepest branch of the Streptophyta in chloroplast genome-based phylogenies. BMC Biology 5: 2.

Lemieux, C., Otis, C. and Turmel, M., 2014. Six newly sequenced chloroplast genomes from prasinophyte green algae provide insights into the relationships among prasinophyte lineages and the diversity of streamlined genome architecture in picoplanktonic species. BMC Genomics 15: 857.

Lemieux, C., Otis, C. and Turmel, M., 2007. A clade uniting the green algae Mesostigma viride and Chlorokybus atmophyticus represents the deepest branch of the Streptophyta in chloroplast genome-based phylogenies. BMC Biology 5: 2.

Liu, Y., Cox, C.J., Wang, W. and Goffinet, B., 2014. Mitochondrial phylogenomics of early land plants: mitigating the effects of saturation, compositional heterogeneity, and codon-usage bias. Systematic Biology 63: 862-878. 
Liu, L., Yu, L. and Edwards, S.V., 2010. A maximum pseudo-likelihood approach for estimating species trees under the coalescent model. BMC Evolutionary Biology 10: 302.

Magallón, S., Hilu, K.W. and Quandt, D., 2013. Land plant evolutionary timeline: gene effects are secondary to fossil constraints in relaxed clock estimation of age and substitution rates. American Journal of Botany 100: 556-573.

Malek, O., Lättig, K., Hiesel, R., Brennicke, A. and Knoop, V., 1996. RNA editing in bryophytes and a molecular phylogeny of land plants. The EMBO Journal 15: 1403-1411.

McCourt, R.M., Delwiche, C.F. and Karol, K.G., 2004. Charophyte algae and land plant origins. Trends in Ecology \& Evolution 19: 661-666.

Mirarab, S., Reaz, R., Bayzid, M.S., Zimmermann, T., Swenson, M.S. and Warnow, T., 2014. ASTRAL: genome-scale coalescent-based species tree estimation. Bioinformatics 30: i541-i548.

Mishler, B.D. and Churchill, S.P., 1984. A cladistic approach to the phylogeny of the "bryophytes". Brittonia 36: 406-424.

Mishler, B.D. and Kelch, D.G., 2009. Phylogenomics and early land plant evolution. In Bryophyte Biology, Eds. Goffinet, B. and Shaw, A.J., $2^{\text {nd }}$ Edition. Cambridge University Press, Cambridge, U.K. Pp.173-197.

Mishler, B.D., Lewis, L.A., Buchheim, M.A., Renzaglia, K.S., Garbary, D.J., Delwiche, C.F., Zechman, F.W., Kantz, T.S. and Chapman, R.L., 1994. Phylogenetic relationships of the" green algae" and" bryophytes". Annals of the Missouri Botanical Garden 81: 451-483.

Nickrent, D.L., Parkinson, C.L., Palmer, J.D. and Duff, R.J., 2000. Multigene phylogeny of land plants with special reference to bryophytes and the earliest land plants. Molecular Biology and Evolution 17: 1885-1895. 
Nishiyama, T. and Kato, M., 1999. Molecular phylogenetic analysis among bryophytes and tracheophytes based on combined data of plastid coded genes and the 18S rRNA gene. Molecular Biology and Evolution 16: 1027-1036.

Nishiyama, T., Wolf, P.G., Kugita, M., Sinclair, R.B., Sugita, M., Sugiura, C., Wakasugi, T., Yamada, K., Yoshinaga, K., Yamaguchi, K. and Ueda, K., 2004. Chloroplast phylogeny indicates that bryophytes are monophyletic. Molecular Biology and Evolution 21: 1813-1819.

Nylander, J.A., Ronquist, F., Huelsenbeck, J.P. and Nieves-Aldrey, J., 2004. Bayesian phylogenetic analysis of combined data. Systematic biology $,=53:$ 47-67.

Patel, S., Kimball, R.T. and Braun, E.L., 2013. Error in phylogenetic estimation for bushes in the tree of life. Journal of Phylogenetics \& Evolutionary Biology 1: 2.

Parenti, L.R., 1980. A phylogenetic analysis of the land plants. Biological Journal of the Linnean Society 13: $225-242$.

Palmer, J.D., Soltis, D.E. and Chase, M.W., 2004. The plant tree of life: an overview and some points of view. American Journal of Botany 91: 1437-1445.

Phillips, M.J., Delsuc, F. and Penny, D., 2004. Genome-scale phylogeny and the detection of systematic biases. Molecular biology and evolution 21: 1455-1458.

Poe, S. and Swofford, D.L., 1999. Taxon sampling revisited. Nature 398: 299.

Puttick, M.N., Morris, J., Williams, T.A., Cox, C.J., Edwards, D., Kenrick, P., Pressel, S., Wellman, C.H., Schneider, H., Pisani, D., Donoghue, P.C.J., 2018. The interrelationships of land plants and the nature of the ancestral embryophyte. Current Biology (in press).

Posada, D. and Crandall, K.A., 1998. Modeltest: testing the model of DNA substitution. Bioinformatics 14: 817-818. 
Qiu, Y.L., Li, L., Wang, B., Chen, Z., Knoop, V., Groth-Malonek, M., Dombrovska, O., Lee, J., Kent, L., Rest, J. and Estabrook, G.F., 2006. The deepest divergences in land plants inferred from phylogenomic evidence. Proceedings of the National Academy of Sciences 103: 15511-15516.

Qiu, Y.L., Li, L., Wang, B., Chen, Z., Dombrovska, O., Lee, J., Kent, L., Li, R., Jobson, R.W., Hendry, T.A. and Taylor, D.W., 2007. A nonflowering land plant phylogeny inferred from nucleotide sequences of seven chloroplast, mitochondrial, and nuclear genes. International Journal of Plant Sciences 168: 691-708.

Quandt, D., Müller, K., Stech, M., Frahm, J.P., Frey, W., Hilu, K.W. and Borsch, T., 2004. Molecular evolution of the chloroplast trnL-F region in land plants. In Molecular Systematics of Bryophytes, Eds. Goffinet, B., Hollowell, V., and Magill, B., Monographs in Systematic Botany from the Missouri Botanical Garden, 98. Missouri Botanical Garden Press, U.S.A. Pp. 13-37.

Renzaglia, K.S., Duff, R.J., Nickrent, D.L. and Garbary, D.J., 2000. Vegetative and reproductive innovations of early land plants: implications for a unified phylogeny. Philosophical Transactions of the Royal Society of London B: Biological Sciences 355: 769-793.

Renzaglia, K.S., Schuette, S., Duff, R.J., Ligrone, R., Shaw, A.J., Mishler, B.D. and Duckett, J.G., 2007. Bryophyte phylogeny: advancing the molecular and morphological frontiers. The Bryologist 110: $179-213$.

Roch, S. and Warnow, T., 2015. On the robustness to gene tree estimation error (or lack thereof) of coalescent-based species tree methods. Systematic Biology 64: 663-676.

Rodríguez-Ezpeleta, N., Philippe, H., Brinkmann, H., Becker, B. and Melkonian, M., 2006. Phylogenetic analyses of nuclear, mitochondrial, and plastid multigene data sets support the placement of Mesostigma in the Streptophyta. Molecular Biology and Evolution 24: 723-731. 
Rokas, A., Williams, B.L., King, N. and Carroll, S.B., 2003. Genome-scale approaches to resolving incongruence in molecular phylogenies. Nature 425: 798.

Rokas, A. and Carroll, S.B., 2005. More genes or more taxa? The relative contribution of gene number and taxon number to phylogenetic accuracy. Molecular Biology and Evolution 22: 13371344.

Ruhfel, B.R., Gitzendanner, M.A., Soltis, P.S., Soltis, D.E. and Burleigh, J.G., 2014. From algae to angiosperms-inferring the phylogeny of green plants (Viridiplantae) from 360 plastid genomes. BMC Evolutionary Biology 14: 23.

Sayyari, E. and Mirarab, S., 2016. Fast coalescent-based computation of local branch support from quartet frequencies. Molecular Biology and Evolution 33: 1654-1668.

Shoemaker, J.S., Painter, I.S. and Weir, B.S., 1999. Bayesian statistics in genetics: a guide for the uninitiated. Trends in Genetics 15: 354-358.

Smith, S.A., Beaulieu, J.M. and Donoghue, M.J., 2009. Mega-phylogeny approach for comparative biology: an alternative to supertree and supermatrix approaches. BMC Evolutionary Biology 9: 37.

Steele, K.P., Holsinger, K.E., Jansen, R.K. and Taylor, D.W., 1991. Assessing the reliability of 5S rRNA sequence data for phylogenetic analysis in green plants. Molecular Biology and Evolution 8: 240-248.

Swofford, D.L., Olsen, G.J., Waddell, P.J. and Hillis, D.M., 1996. Phylogenetic inference. In Molecular systematics, Eds. Hillis, D.M., Moritz, C. and Mable, B.K. Sinauer Associates, Sunderland, Massachusetts, U.S.A.

Szöllősi, G.J., Tannier, E., Daubin, V. and Boussau, B., 2014. The inference of gene trees with species trees. Systematic Biology 64: 42-62. 
Timme, R.E., Bachvaroff, T.R. and Delwiche, C.F., 2012. Broad phylogenomic sampling and the sister lineage of land plants. PLoS One 7: e29696.

Turmel, M., Otis, C. and Lemieux, C., 2006. The chloroplast genome sequence of Chara vulgaris sheds new light into the closest green algal relatives of land plants. Molecular Biology and Evolution 23: 1324-1338.

Turmel, M., Otis, C. and Lemieux, C., 2013. Tracing the evolution of streptophyte algae and their mitochondrial genome. Genome Biology and Evolution 5: 1817-1835.

Waters, D.A., Buchheim, M.A., Dewey, R.A. and Chapman, R.L., 1992. Preliminary inferences of the phylogeny of bryophytes from nuclear-encoded ribosomal RNA sequences. American Journal of Botany 79: 459-466.

Whitfield, J.B. and Lockhart, P.J., 2007. Deciphering ancient rapid radiations. Trends in Ecology \& Evolution 22: 258-265.

Wickett, N.J., Mirarab, S., Nguyen, N., Warnow, T., Carpenter, E., Matasci, N., Ayyampalayam, S., Barker, M.S., Burleigh, J.G., Gitzendanner, M.A., Ruhfel, B.R., Wafula, E., Der, J.P, Graham, S.W., Mathews, S., Melkonian, M., Soltis, D.E., Soltis, P.S., Miles, N.W., Rothfels, C.J., Pokorny, L., Shaw, A.J., DeGironimo, L., Stevenson, D.W., Barbara, S., Villarreal, J.C., Roure, B., Philippe, H., dePamphilis, C.W., Chen, T., Deyholos, M.K., Baucom, R.S., Kutchan, T.M., Augustin, M.M., Wang, J., Zhang, Y., Tian, Z., Yan, Z., Wu, X., Sun, X., Wong G.K-S., Leebens-Mack, J., 2014. Phylotranscriptomic analysis of the origin and early diversification of land plants. Proceedings of the National Academy of Sciences 111: 4859-4868.

Wodniok, S., Brinkmann, H., Glöckner, G., Heidel, A.J., Philippe, H., Melkonian, M. and Becker, B., 2011. Origin of land plants: do conjugating green algae hold the key? BMC Evolutionary Biology, 11: 104. 
Wolf, P.G., Karol, K.G., Mandoli, D.F., Kuehl, J., Arumuganathan, K., Ellis, M.W., Mishler, B.D., Kelch, D.G., Olmstead, R.G. and Boore, J.L., 2005. The first complete chloroplast genome sequence of a lycophyte, Huperzia lucidula (Lycopodiaceae). Gene 350: 117-128.

Xu, B., Ohtani, M., Yamaguchi, M., Toyooka, K., Wakazaki, M., Sato, M., Kubo, M., Nakano, Y., Sano, R., Hiwatashi, Y. and Murata, T., 2014. Contribution of NAC transcription factors to plant adaptation to land. Science 343: 1505-1508.

Yang, Z., 1994. Maximum likelihood phylogenetic estimation from DNA sequences with variable rates over sites: approximate methods. Journal of Molecular Evolution 39: 306-314.

Zhong, B., Xi, Z., Goremykin, V.V., Fong, R., Mclenachan, P.A., Novis, P.M., Davis, C.C. and Penny, D., 2013. Streptophyte algae and the origin of land plants revisited using heterogeneous models with three new algal chloroplast genomes. Molecular Biology and Evolution 31: 177-183. 


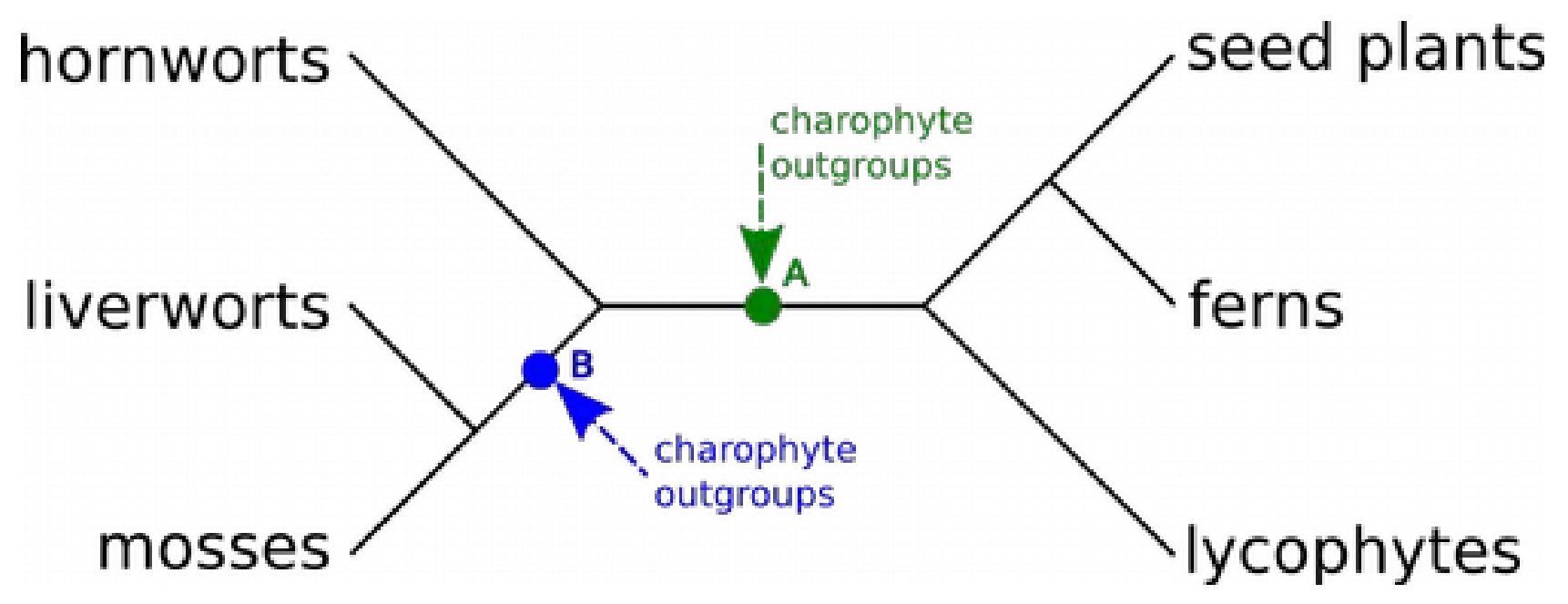

Figure 1. Unrooted phylogeny of land plants. The unrooted relationship among land plants are well supported by recent studies. Indicated on the phylogeny are the two most probable rooting positions of the charophyte algal outgroups as argued in these analyses: A) monophyletic bryophytes, B) paraphyletic bryophytes, with liverwort plus mosses-basal, and hornworts the sistergroup to tracheophytes. 